\title{
METHODOLOGICAL CONCEPTS OF BUILDING UP THE CREATIVITY TYPOLOGY
}

\author{
Diana Bogoyavlenskaya \\ Psychological Institute of Russian Academy of Education, Russia \\ Liubov Kotlyarova \\ All-Russian State University of Justice, Russia
}

\begin{abstract}
The article traces the historical dynamics of the initial concepts of creativity. It is shown how the concepts of Russian methodology provided a principally new approach to studies of creativity psychology and building up the creativity typology. The original method of the Creative Field allows defining stimulus-productive, heuristic and creative types of the approaches to creativity. The conditions of carrying out the experiment using this method are described.
\end{abstract}

Further the research results are given referring characteristics of the motivational profile in groups, defined according to the creativity typology.

Keywords: creativity, analysis unit, the Creative Field method, motivational profile.

\section{Introduction}

The concept of creativity is one of scientific paradoxes. Along with the fact that it characterizes a phenomenon of genus essence of a human, it still does not have a whole clarification not only in mass cognition, but also in scientific literature. Popular definition of creativity as a creation of something new does not contradict common sense, but characterizes it only by its product neglecting the nature of the process itself. In the same time it justifies absence of differentiation of wide, heterogeneous phenomenology of creativity, transferring all its types in the same plane, leveling their differences and diluting the concept of specifics of creativity as it is.

Genial discoveries advancing contemporary science for ages and solving a problem by a schoolboy are still considered in the same plane. To creativity belongs building up a scientific theory along with solving of a puzzle.

Experimental research of creativity leaving the concept of cognition as reproductive process within the framework of associationism and reaching its understanding as productivity in gestalt-psychology could not move further. An insight providing solving problem situations could appeal to intellect only, 
making it equal with creativity. Thus, creativity was defined merely as intellect level. That led to the situation when creative abilities were diagnosed using tests with a set of tasks for presence of intellectual operations or solving "creative problems". The tendency was strengthened by the requirements of psychometric paradigm, which Stern defined with maximum clearance: "The need of measurement leads to narrowing of a concept" (Stern, 1997). Thus, understanding the concept of creativity was based on a technique of measuring certain elements. This fact underlined the tendency which Vygotsky named "element-wise analysis", i.e. reducing the whole to one component. But "on the way of identification of the whole with an element, a problem is not solved, it is just passed over" (Vygotsky, 1982, p. 13).

A crucial factor that defined the direction of research of these phenomena was Vygotsky's methodological directive on two ways of scientific development. This is a direct way of complication of a structure and its functions, which Nature took; and a reverse way outgoing from knowledge of the highest form. The direct way comes to describing a psychic phenomenon, basing on concrete empirically established by any possible way characteristics. Due to the absence of exact criteria for the highest form all the forms can be considered as having equal significance. We can see it nowadays when we accept that there is a variety of concepts of giftedness and creativity. Either an insight (in gestalt-psychology) or divergent thinking (Guilford, 1959) or perhaps association by similarity (James, 1950) provides a flash of inspiration of a genius. Kudriavtsev (2015) uses the concept of "potentiation" (Schelling's term) as growth of child's abilities. And the whole phenomenology of productive thinking is creativity. And each one has his own truth.

An opposite way is based on a theoretical definition of a higher form reflecting the essence of the under study phenomenon. However, the analysis of the highest form is important for identifying the simplest form, which according to Aristotle reflects the essence of analysis (analysis unit). In his appeal to psychology willing to study complex phenomena Vygotsky demands to change methods of resolution to methods of analysis that isolates units. This approach provides finally the way of understanding of creativity nature not by its product, but by its mechanism.

Francis Galton came closer to the point than the others as he mentioned devotion to a cause as a characteristic feature of gifted persons, but he did not make the final step (and in was not possible at that moment). We managed to justify theoretically and methodologically the phenomena he noticed empirically.

The objective of studying the action losing a form of response demands designing a new model of experiment, opposite to the methods built by the "stimulus-reaction" principle, "a challenge by our self-will" (Chelpanov, 1999). 
Only on the way of realization of Vygotsky's methodological principles it became possible to define the mechanism of creation of new forms. It is on the way of defining and theoretical justifying the highest form of creativity where we consider creativity phenomena, losing the form of response. These are "porism" (a term introduced by ancient Greeks) as an unforeseen going out into "not-specified" and types of discoveries described by Claparède (2007) and by Hadamard (1945). Those are discoveries that are not connected with solving of given tasks, but unexpected discovery of a new fact. For studying this kind of creativity a new type of experimental model had to be built up. In its turn, this new model allowed us to introduce a new psycho-diagnostics method for convenience called The Creative Field.

An experiment using this method allowed identifying the creativity analysis unit, which is the ability of a person to develop the accepted activity by one's initiative. Basing on this creativity analysis unit first creativity typology was built up, i.e. the differentiation of the whole heterogeneous phenomenology of creativity was carried out (Bogoyavlenskaya, 2009).

\section{The model of experiment}

The estimation of personal potential within a certain time period requires space for following train of thought outside the limitations of solution of the initial problem. A new experimental model should be represented by the activity which is variable, but in a different way comparing with tests including a set of various tasks. It has to be homogeneous and to have differences at the same time. The system of problems of the same type meets these requirements. They are solved the same way, but differ by a certain parameter. Thus, we can watch the process of mastering an activity and at the first stage estimate mental abilities of a testee by characteristics of one's educability and all its indexes (rate of advancing, level of generality, consciousness, independence). That allows overcoming imperfection of testing, defining intellect more and more precisely (Bogoyavlenskaya, 2009).

The next stage of the experiment allows following the process after mastering activity and identifying the phenomenon of creativity itself as the going out the limitations of given situation. Since the system of single-type problems has a range of common patterns, it provides building up a two-layer model of activity. The first, surface layer is the given activity consisting of solving of certain problems. The second, subsurface layer (masked by the first layer and not obvious for a testee) is the activity of defining hidden patterns, included into system of problems. The discovering of those is not needed for direct problem solving, thus, the space for observing the activity development process is created. 


\section{The Creative Field method}

The studying of a principle which looses the shape of response requires the realization of the first principle - absence of outer and inner evaluative stimuli. It becomes possible due to the fact that the second layer is not given explicitly in the experimental situation, but is kept there surreptitiously or implicitly. The richer this layer of activity is the wider is the system of patterns, the more discrete is their hierarchy, the more diagnostic and prognostic value has a certain diagnostic technique.

Since the abilities of a testee can reveal themselves only in situation of overcoming and leaving the bounds of the initial situation, the "ceiling" (a limitation connected with a certain way of solving a problem) is given but it has to be overcome or withdrawn. The structure of the experimental material should provide a system of such false, apparent "ceilings", but the system itself should have no limits. This is its difference from "open" problems. Thus, the second principle lies in the requirement of absence of a "ceiling" which restricts the activity in whole.

Full-fledged, methodologically consistent realization of these principles is possible only if testee's activity is not limited by time frame and if the experiment is repeated (the third principle).

We would like to emphasise that a long-time research of the same sample has lead us to the conclusion that the studying of creativity should be multiple and be carried out in the conditions not limited by any time frame.

Besides, in order to provide real durability and recurrence of studies, the activity should be variable, permanent and heterogeneous on various stages of the experiment.

The above-mentioned principles create a method which was called the Creative Field for convenience. The realization of this technique is possible only on the assumption of fulfilment of the principles in their unity as well as by individual testing, whereas by group (mass) testing as Chelpanov stated in the beginning of the last century "the individuality of each testee is lost, originality of mental process running in his mind is concealed. Facts to be essential for conclusions are wasted" (Chelpanov, 1999, p. 368).

\section{The Typology of Creativity}

The technique of the Creative Field allows marking out various levels of performance and differentiating the whole complex and heterogeneous phenomenology of creativity. This concept provides designing of typology of creativity according to cognition levels. 
The activities stimulated by outer factors (including high level of performance) belong to the first type called "stimulus-productive". The cognition process of this level focuses on the certain situation and is performed on the level of single, according to the philosophical classification. The major part of mankind belongs to this type.

The activity that is developed by the person's initiative belongs to the second type - "heuristic". This is the level of art and laws discovering, which was referred by Rubinstein (1935) as "explosion of layers of things in existence". This is the cognitive process on the level of special. It is gift that is characterized that way in the philosophic literature.

The last level - the creative - is characterized not only by discovering new patterns, but by their theoretical proving as well. It is the level of building up theories and defining new problems. The cognitive process is performed on the level of the whole. Such process provides cognition of the essence of an object. Furthermore, having known the essence of a phenomenon, one can predict qualitative leaps in its development and this characterizes the prognostic abilities of a subject. According to philosophers, it is this ability that more than anything defines a genius who predicts future on hundreds of years ahead.

Thus, high indexes on the first (stimulus-productive) level prove just high intellectual abilities of a subject. Last two levels (heuristic and creative) identify creative abilities, i.e. depth of cognition. The necessity of marking two levels explains using the term "creative", which in this context is an alternative of understanding of creativity as divergent productivity.

Alongside with that the ability to develop activity by one's initiative can be explained just by the characteristics of intellect. It is a quality of integrated personality, reflecting the interaction of cognitive and emotional spheres in their unity, where abstraction of one of the sides is impossible, as they are inseparable. That "alloy" of capabilities and personality possesses a quality of generality, i.e. belongs to the whole as to the unity and corresponds to the methodological requirements to the analysis unit of the creativity.

The validity of the Creative Field method is proved in several cycles of research supervised by Diana B. Bogoyavlenskaya. The results of the research are presented in previously published works. In the same time an issue of the characteristics of motivational sphere of adults belonging to various creativity types (defined by the Creative Field method) remains little-studied.

\section{Research aim and methods}

The aim of the current research is studying motivational characteristics reflecting to main personality orientations (consuming and productive) in groups, defined by the Creative Field method. 
For reaching this aim the following objectives are settled: (1) marking out the typological groups by the Creative Field method; (2) defining the typology of motivational structure in each group.

For studying motivational structure the Milman's technique the Diagnostics of Personal Motivational Structure (further on - DPMS) is used (Milman, 2005). According to the author's conception the motivational scales reflect the main personal orientations - consuming and productive.

The DPMS technique includes seven motivational scales. For the completeness of diagnostics of personal motivational sphere each scale is divided to four more scales, reflecting: whole-life motivation (concerning the whole sphere of life activity); working (learning) motivation; "ideal" state of the motive understood by the author as "a level of a drive to act"; real state - the degree of satisfaction of a certain motive at present as well as forces applied for reaching satisfaction of this motive.

The total index of personal motivational sphere according to the testing results consists of 28 scales of motivational profile. If needed, various scales can be consolidated. The whole personal motivational picture is reflected in a personal motivational profile representing in quantitative or graphical form the correlations between various motivational scales, registered by a psychodiagnostic method. A character of motivational profile (MP) can be defined according to the profiles typology. After defining the characteristics of motivational sphere of each testee, his/her motivational profile can be attributed to one of the following types: progressive, regressive, expressive, impulsive, flat or combined.

The obtained data were processed by mathematics and statistics methods; the comparative analysis was applied.

\section{Research design and sample characteristics}

The research was carried out in 2016. The research sample consisted of university students $(\mathrm{N}=135$, age 19-20 years). The participation in the research was completely voluntary without using any motivational factors. The meetings with participants took place in social-psychological service facilities, in assessment centres. The specialists of the services were engaged in the research procedures after completing a special training related the research techniques.

\section{Research results}

Let us consider the results obtained by using of the Creative Field technique.

According to the results obtained by using this method three groups of participants are defined, corresponding to three creativity types: stimulusproductive $(\mathrm{SP})$, heuristic $(\mathrm{H})$ and creative $(\mathrm{C})$. 
As mentioned above, the Creative Field method allows defining three qualitative creativity types: stimulus-productive, heuristic and creative. In case of participant's rejection of a task after its introduction, his/her creative activity is considered as low-productive.

In the process of research there are respondents who are agree to participate in the research, but refuse it after introduction of the problems (of even after having solved a part of them). This group is marked is the N-group.

The following distribution according to the creativity type is obtained: stimulus-productive type $-61.5 \%$ (SP group), heuristic type $-8.5 \%$ (H group), creative $-1.5 \%$ (Cr-group).

Such distribution corresponds in general to the results obtained by other researchers in samples of adults (Bogoyavlenskaya \& Bogoyavlenskaya, 2013). Those data show that the majority of people belong to the stimulus-productive type.

The next objective of the research is defining motivational profile type in the groups of stimulus-productive, heuristic and creative type. At first, the motivational profile type of every participant is defined, and then the distribution (percentage) of motivational profiles in each group is described.

In the SP-group the following distribution of motivational profiles types is found: progressive $-7 \%$, regressive $-18 \%$, expressive $-63 \%$, impulsive $-5 \%$, flat $-0 \%$, progressive-expressive (combined) $-7 \%$, progressive-impulsive $-0 \%$, regressive-impulsive $-0 \%$, and regressive-expressive $-0 \%$.

The expressive type related to self-affirmation in the society, developed ambition, eccentricity, a tendency to a constant rising of aspiration level, appears to dominate in this group.

In the H-group (heuristic type of creativity) the distribution of motivational profile type is following: progressive $-49.6 \%$, regressive $-11 \%$, expressive $13.4 \%$, impulsive $-5 \%$, flat $-0 \%$, progressive-expressive (combined) $-11 \%$, progressive-impulsive $-5 \%$, regressive-impulsive $-5 \%$, and regressiveexpressive $-0 \%$. As it is seen in the distribution the progressive type of motivational profile dominates in this group. In accordance with Milman's ideas, such type of profile positively correlates with "successful working and studying activities. Most often this type of the MP is met among creative people... At the same time, this kind of profile is typical for a socially-oriented person and that fact can be included into a concept of creative and productive orientation of a person" (Milman, 2005, p. 42).

In the Cr-group (the creative type) which includes just 2 participants of the research, the only type of the motivational type dominates - the progressiveexpressive one. Taking into the account the small number of participants in this group it is impossible to make a conclusion on the firm correlations in the group. 
Diana Bogoyavlenskaya, Liubov Kotlyarova. Methodological Concepts of Building up the Creativity Typology

In the $\mathrm{N}$-group the following types of motivational profiles are marked out: progressive $-0 \%$, regressive $-39.2 \%$, expressive $-14.5 \%$, impulsive $-8.2 \%$, flat $-0 \%$, progressive-expressive $-3.7 \%$, progressive-impulsive $-1.48 \%$, regressive-impulsive $-14.5 \%$, regressive-expressive $-4.4 \%$. The tendency to domination of the regressive profile should be mentioned. The regressive type of profile is characterized by exceeding of general level of consuming motives above productive or developing ones.

\section{Conclusion}

Summarizing the obtained data, on our opinion, it is possible to make a conclusion on the existence of stable connections between personal motivational orientation and creative activity. In the research it is shown that the persons with heuristic type of creative activity have a pronounced motivation aimed at development of the activity by one's initiative and that identifies its creative character.

In the group of stimulus-productive type dominates the expressive type of the motivational profile, related to the tendency of self-affirmation in the society, developed ambition, eccentricity and a tendency to a constant rising of aspiration level.

The presented theoretical approach is believed to have an important meaning for educational practice.

\section{References}

Bogoyavlenskaya, D. B. (2009). Psychology of creative abilities. Samara: ID Fedorov. [in Russian].

Bogoyavlenskaya, D. B., \& Bogoyavlenskaya, M. E. (2013). Giftedness: concept and diagnostics. Moscow: Razvitie lichnosti. 2013 [in Russian]. [in Russian].

Claparède, E. (2007). Psychology of a child and experimental pedagogies. URSS. [in Russian].

Chelpanov, G. I. (1999). Psychology, philosophy, education. Selected works. Voronezh. [in Russian].

Guilford, J. P. (1959). Three faces of intellect. American Psychologist. Vol. 14 / 8

Hamaragd, J. (1945). An Essay on the Psychology of Invention in the Mathematical Field. Princeton University Press.

James, W. (1950). The Principles of Psychology. Dover Publications.

Kudriavtsev, V. T. (2013). Developing education: contituity of pre-school and primary school. Vestnik. Vol. 11. [in Russian].

Milman, V. E. (2005). Motivation of creativity and growth: Structure, diagnostics, development. Moscow, Mireya and Co. [in Russian].

Rubenstein, S. L. (2015). Basics of general psychology. StPetersburg, Peter. [in Russian].

Stern, W. (1997). Mental giftedness. Psychological methods of mental giftedness testing and their application to the students of the school age. Moscow, Souyuz. [in Russian].

Vygotsky, L.S. (1982). Thinking and Speech. Moscow, Pedagoguika. [in Russian]. 